\title{
Frequent detection of bocavirus DNA in German children with respiratory tract infections Benedikt Weissbrich*1, Florian Neske ${ }^{1}$, Jörg Schubert ${ }^{1}$, Franz Tollmann ${ }^{1}$, Katharina Blath ${ }^{1,2}$, Kerstin Blessing ${ }^{2}$ and Hans Wolfgang Kreth ${ }^{2}$
}

\author{
Address: ${ }^{1}$ Institute of Virology and Immunobiology, University of Würzburg, Versbacher Str. 7, 97078 Würzburg, Germany and ${ }^{2}$ Children's \\ Hospital, University of Würzburg, Josef-Schneider-Str. 2, 97080 Würzburg, Germany \\ Email: Benedikt Weissbrich* - weissbrich@vim.uni-wuerzburg.de; Florian Neske - florian.neske@gmx.de; Jörg Schubert - schubert@vim.uni- \\ wuerzburg.de; Franz Tollmann - tollmann@vim.uni-wuerzburg.de; Katharina Blath - blathk@web.de; \\ Kerstin Blessing - blessing_k@kinderklinik.uni-wuerzburg.de; Hans Wolfgang Kreth - kreth@mail.uni-wuerzburg.de \\ * Corresponding author
}

Published: II July 2006

BMC Infectious Diseases 2006, 6:109 doi:10.1186/1471-2334-6-109
Received: II April 2006

Accepted: II July 2006

This article is available from: http://www.biomedcentral.com/I47/-2334/6/109

(C) 2006 Weissbrich et al; licensee BioMed Central Ltd.

This is an Open Access article distributed under the terms of the Creative Commons Attribution License (http://creativecommons.org/licenses/by/2.0), which permits unrestricted use, distribution, and reproduction in any medium, provided the original work is properly cited.

\begin{abstract}
Background: In a substantial proportion of respiratory tract diseases of suspected infectious origin, the etiology is unknown. Some of these cases may be caused by the recently described human bocavirus (hBoV). The aim of this study was to investigate the frequency and the potential clinical relevance of $\mathrm{hBoV}$ in pediatric patients.
\end{abstract}

Methods: We tested 835 nasopharyngeal aspirates (NPA) obtained between 2002 and 2005 from pediatric in-patients with acute respiratory tract diseases at the University of Würzburg, Germany, for the presence of hBoV DNA. The specificity of positive PCR reactions was confirmed by sequencing.

Results: HBoV DNA was found in 87 (10.3 \%) of the NPAs. The median age of the infants and children with $\mathrm{hBoV}$ infection was 1.8 years (mean age 2.0 years; range 18 days -8 years). Infections with $\mathrm{hBoV}$ were found year-round, though most occurred in the winter months. Coinfections were found in $34(39.1 \%)$ of the hBoV positive samples. RSV, influenza A, and adenoviruses were most frequently detected as coinfecting agents. Sequence determination of the PCR products in the NPI region revealed high identity ( $99 \%$ ) between the nucleotide sequences obtained in different years and in comparison to the Swedish viruses STI and ST2. An association of hBoV with a distinct respiratory tract manifestation was not apparent.

Conclusion: $\mathrm{HBOV}$ is frequently found in NPAs of hospitalized infants and children with acute respiratory tract diseases. Proving the clinical relevance of $\mathrm{hBoV}$ is challenging, because application of some of Koch's revised postulates is not possible. Because of the high rate of coinfections with $\mathrm{hBoV}$ and other respiratory tract pathogens, an association between $\mathrm{hBoV}$ and respiratory tract diseases remains unproven. 


\section{Background}

Respiratory tract infections are a major cause of human morbidity and are caused by a broad spectrum of microbial agents. Viruses account for the largest number of respiratory tract infections. The so-called respiratory viruses include influenza virus $\mathrm{A}$ and $\mathrm{B}$, parainfluenzae viruses, adenoviruses, respiratory syncytial virus (RSV), rhinoviruses, and coronaviruses. In recent years, several novel viruses have been discovered in patients with respiratory infections using molecular biology methods. These novel viruses include the human metapneumovirus and several coronaviruses (SARS, NL63, HKU1) [1,2]. The latest addition to this list was the human bocavirus (hBoV) described by Allander et al. [3]. Their screening method for unknown viral sequences in patient samples involved concentration of viral particles, nucleic acid isolation, random amplification of RNA and DNA, and finally sequencing and subsequent blasting of the amplified products. $\mathrm{HBoV}$ is most closely related to the minute virus of canines (MVC) and the Bovine Parvovirus (BPV), which have been classified in the genus Bocavirus within the Parvoviridae [4].

Classically, the postulates of Koch as modified by Rivers have been used to establish a causal relationship between viruses and a disease [5]. However, hBoV has not been propagated in cell culture, and there is no animal model so far. Therefore, proving the clinical relevance is challenging because application of some postulates of Koch and Rivers is not possible.

In the first description of hBoV, DNA was detected in 17 $(3.1 \%)$ of 540 Swedish children with lower respiratory tract disease. Three of the children were coinfected with other viruses (two with RSV and one with adenovirus). Because $14 \mathrm{hBoV}$-positive samples were negative for other respiratory viruses by standard screening, it was reasoned that hBoV may cause respiratory tract disease. In a second report of hBoV in Australian children and adults, hBoV was detected in 18 (5.6\%) of 324 respiratory samples. In ten of these $(55.6 \%)$, a coinfection with RSV $(n=8)$, hMPV or adenoviruses was detected [6]. In a third study from Japan, hBoV DNA was found in 18 (5.7 \%) of 318 respiratory specimens of children with lower respiratory tract disease [7]. Samples positive for other viruses (RSV, influenza virus $A$ and $B, h M P V)$ were excluded from the study. Analysis of double infections was therefore not possible. In two more recent studies from Canada and France, $\mathrm{hBoV}$ DNA was detected in 18 of $1209(1.5 \%)$ and nine of $262(3.4 \%)$ respiratory samples, respectively $[8,9]$. In the Canadian study specimens of children and adults were tested. Specimens positive for other respiratory viruses were excluded. The French study examined samples of children below five years of age. Three of the nine hBoV DNA positive children (33.3 \%) were coinfected with RSV.
In order to better understand the epidemiological pattern of $\mathrm{hBoV}$ infections and to analyze its clinical relevance, further studies on larger groups of patients are necessary. Therefore, we retrospectively tested nasopharyngeal aspirates of patients from the University of Würzburg Children's Hospital, Germany for the presence of hBoV DNA.

\section{Methods \\ Samples}

The samples tested for hBoV infection consisted of stored nasopharyngeal aspirates (NPA) that were sent by the University of Würzburg Children's hospital for screening of respiratory viruses from January 2002 to September 2005. On arrival in the viral diagnostic laboratory, the samples were routinely tested for the presence of respiratory virus antigens with an immunofluorescence assay (Respiratory Panel IFA Kit, Chemicon). The screening reagent of the kit detects antigens of adenoviruses, influenza viruses $A$ and $B$, parainfluenza viruses $1-3$, and RSV. NPAs showing positive reactions with the screening reagent were further studied by IFA using the seven single monoclonal antibodies contained in the screening reagent. Remaining NPA material was stored at $-20^{\circ} \mathrm{C}$ until further testing for $\mathrm{hBoV}$ DNA. In addition to the samples from $2002-2005$, a small number of samples $(\mathrm{n}=17)$ from 1997 - 2001 were also available for retrospective testing. The study was carried out in compliance with the Helsinki declaration and was approved by the ethics committee of the medical faculty at the University of Würzburg.

\section{hBoV PCR and sequencing}

DNA was extracted from $200 \mu \mathrm{l}$ of the NPA samples using the High Pure Viral Nucleic Acid Kit (Roche, Mannheim, Germany) according to the instructions of the manufacturer. The elution volume was $50 \mu \mathrm{l}$. Amplification of $\mathrm{hBoV}$ DNA was performed with the NP-1 primers BoV188F (GAGCTCTGTAAGTACTATTAC) and BoV542R (CTCTGTGTTGACTGAATACAG) described by Allander [3] using the HotStarTaq DNA Polymerase (Qiagen, Hilden, Germany). PCR reactions were carried out in a 50 $\mu \mathrm{l}$ volume consisting of $5 \mu \mathrm{l}$ extracted DNA, $1 \times$ Qiagen HotStar buffer, dNTPs at a final concentration of $200 \mu \mathrm{M}$ each, 200 pmol of each primer, and $1.5 \mathrm{U}$ of Taq Polymerase. The cycling conditions were 50 cycles $\left(94^{\circ} \mathrm{C} 30 \mathrm{~s}\right.$, $53^{\circ} \mathrm{C} 40 \mathrm{~s}$ and $1 \mathrm{~min}$ at $72^{\circ} \mathrm{C}$ ) after a preheating step of 10 min at $95^{\circ} \mathrm{C}$.

After amplification, PCR products were visualized by staining with ethidium bromide on agarose gels. A PCR reaction was considered as positive when a band of the expected size ( 354 base pairs) was visible. To confirm the sequence specificity, all PCR products from positive reactions were sequenced completely in both directions using 
Big Dye terminator chemistry and the ABI Prism 3100 (Applied Biosystems, Darmstadt, Germany).

General laboratory procedures to prevent PCR contamination were strictly adhered to. One negative control was extracted and amplified for every five NPA samples. All negative controls were found to be negative for $\mathrm{hBoV}$ DNA. A plasmid containing the PCR product cloned in the vector $\mathrm{pCR}^{\circledast} 2.1-\mathrm{TOPO}^{\circledast}$ (Invitrogen) was used as positive control. The sensitivity of the hBoV assay was approximately 10 copies per reaction.

\section{Results}

From January 2002 to September 2005, 901 nasopharyngeal aspirates (NPA) of 786 hospitalized infants and children with febrile respiratory tract diseases were received for viral diagnostic evaluation. The median age of the patients was 1.6 years (mean age 3.4 years; range 12 days - 16 years), and $59 \%$ were boys. The seasonal distribution of all samples is shown in Figure 1. Because of insufficient volume of the stored material, 66 samples had to be excluded from the retrospective testing of hBoV DNA. The median age and the seasonal distribution of the hospitalization were not significantly different between the patients with and without sufficient NPA sample volume.

By routine immunofluorescence testing for antigen of respiratory viruses, a positive diagnosis was obtained for 357 $(39.5 \%)$ of the NPA samples. RSV $(n=158 ; 17.4 \%)$ and influenza A virus $(\mathrm{n}=98 ; 10.9 \%)$ were most frequently found. Further details are shown in Table 1. Coinfections with two or more viruses were detected in 7 of the cases using the antigen assay.

Of the 835 NPAs tested for hBoV DNA, 87 (10.3\%) samples were found to be positive by PCR and subsequent sequencing. The male to female ratio of the $\mathrm{hBoV}$ positive infants and children ( $59.8 \%$ boys) was similar to the ratio in the population tested. Their median age was 1.8 years (mean 2.0 years; range 18 days -8 years). The age distribution of the $\mathrm{hBoV}$ positive patients and of the RSV, adenovirus and influenza $A$ virus positive children for comparison is shown in Figure 2. Median ages were significantly different for these four infectious agents, except for the comparison between influenza $\mathrm{A}$ and adenovirus infections (Table 2). While infections with RSV peaked during the first six months of life (median 0.7 years), most hBoV infections $(\mathrm{n}=52 ; 59.8 \%)$ occurred at the age of 1 - 3 years. Infections with influenza A virus were more evenly distributed over a wider age range (median 2.7 years).

Infections with $\mathrm{hBoV}$ were found year-round, though most occurred in the winter months (Figure 1). The shape of the curves of the total number of NPA samples received and of the hBoV positive samples appeared to be almost in parallel. There were no significant differences between the yearly frequencies of $\mathrm{hBoV}$ positive results between 2002 and 2005 (Table 3). In 17 samples that were available from before 2002, HBoV was retrospectively found in 3 samples, two from 1998 and one from 2001.

In $34(39.1 \%)$ of the hBoV positive infants and children, coinfections with other respiratory viruses were present, most frequently with RSV $(\mathrm{n}=14)$ followed by influenza A $(n=9)$. The percentage distribution of the coinfecting agents among the $\mathrm{hBoV}$ positive samples was similar to the distribution of these agents in the total population (Table 1).

Sequence determination of the hBoV PCR products (NP-1 region of the genome) revealed high identity (99\%) between the nucleotide sequences obtained in different years and in comparison to the Swedish viruses ST1 and ST2.

Clinical data were available for 63 of the $87 \mathrm{hBoV}$ positive NPAs. The patients suffered from upper and/or lower respiratory tract diseases (Table 4). Associations between all $\mathrm{hBoV}$ infections and distinct clinical manifestations were not apparent. Because of the retrospective nature of the study and because clinical data for the $\mathrm{hBoV}$ negative samples were not obtained, a statistical analysis of this aspect was not possible. When the clinical diagnoses of the children with and without coinfections with other respiratory pathogens were compared, pneumonia was found more often in the group of children without coinfections (Table 4). This association was borderline significant ( $p=0.044$ by Fisher's exact test).

\section{Discussion}

We found hBoV DNA in $10.3 \%$ of NPA samples obtained from infants and children with respiratory tract diseases during the years 2002 to 2005 in the region of northern Bavaria in Germany. This is the highest frequency reported so far. To our knowledge, there have been five previous reports on $\mathrm{hBoV}$ infections, the original report from Sweden with a frequency of $3.1 \%$, and reports from Australia, Japan, Canada and France with frequencies of $5.6 \%, 5.7 \%, 1.5 \%$, and $3.4 \%$, respectively. In our study, $\mathrm{hBoV}$ infections were almost as frequently found as infections with influenza A virus, the second most common respiratory infection, and they were considerably more frequent than infections with influenza B, parainfluenzae, and adenoviruses. However, in contrast to the PCR method used for hBoV detection, the other respiratory viruses in our study were examined by IFA. When comparing the detection frequencies, this difference in detection methods has to be taken into consideration. In general, PCR assays are more sensitive than antigen detection 


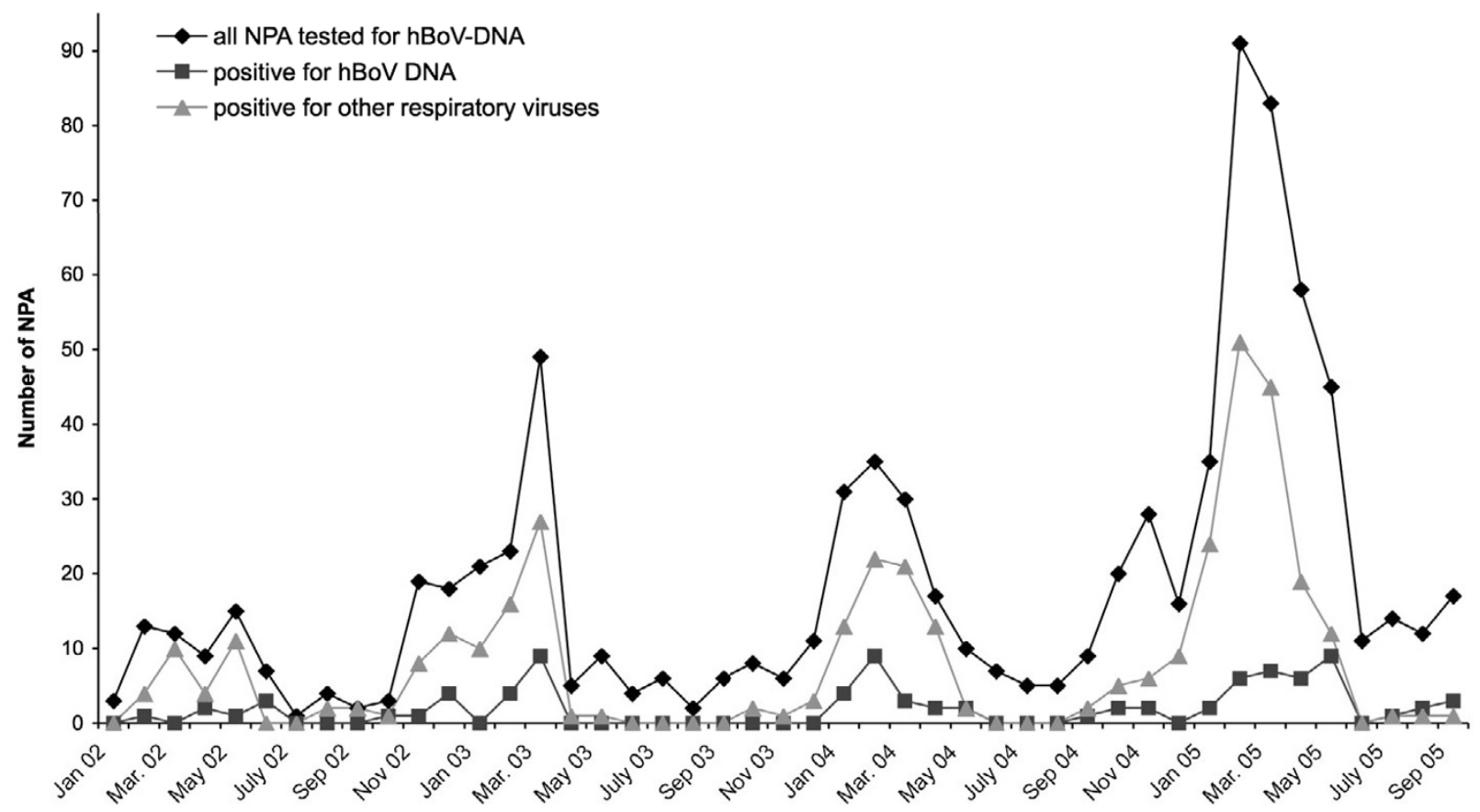

Figure I

Temporal distribution of $\mathrm{hBoV}$ infections. Temporal distribution of the $\mathrm{hBoV}$ DNA positive NPAs compared with the total number of NPAs received and with the total number of positive results by immunofluorescence staining for antigen of respiratory viruses (RSV, influenza $A / B$, parainfluenza $I / 2 / 3$, adenoviruses).

Table I: Frequency of detection of respiratory viruses and of hBoV in NPA samples

\begin{tabular}{|c|c|c|c|}
\hline & Total population $\mathrm{n}$ & Positive results $n$ & Males \\
\hline $\begin{array}{l}\text { Results of IFA testing for viral } \\
\text { antigen }\end{array}$ & 901 & & $59.0 \%$ \\
\hline Total positive results by IFA & 901 & $357(39.5 \%)$ & $55.5 \%$ \\
\hline - RSV & 901 & $158(17.4 \%)$ & $52.5 \%$ \\
\hline - Influenza A & 901 & $98(10.9 \%)$ & $57.1 \%$ \\
\hline - Adenovirus & 901 & $53(5.9 \%)$ & $62.3 \%$ \\
\hline - Parainfluenza I/2/3 & 901 & $39(4.3 \%)$ & $48.7 \%$ \\
\hline - Influenza B & 901 & $16(1.8 \%)$ & $50.0 \%$ \\
\hline - Coinfections & 901 & $7(0.8 \%)$ & $14.3 \%$ \\
\hline Results of hBoV PCR & 835 & $87(10.3 \%)$ & $59.8 \%$ \\
\hline hBoV positive with coinfections & 87 & 34 (39.1 \%) & $58.8 \%$ \\
\hline • with RSV & 87 & $14(16.1 \%)$ & \\
\hline - with influenza $A$ & 87 & $9(10.3 \%)$ & \\
\hline - with adenovirus & 87 & $9(10.3 \%)$ & \\
\hline - with parainfluenza $1 / 2 / 3$ & 87 & I (I.I \%) & \\
\hline - with influenza B & 87 & $\mathrm{I}(\mathrm{I} .1 \%)$ & \\
\hline
\end{tabular}




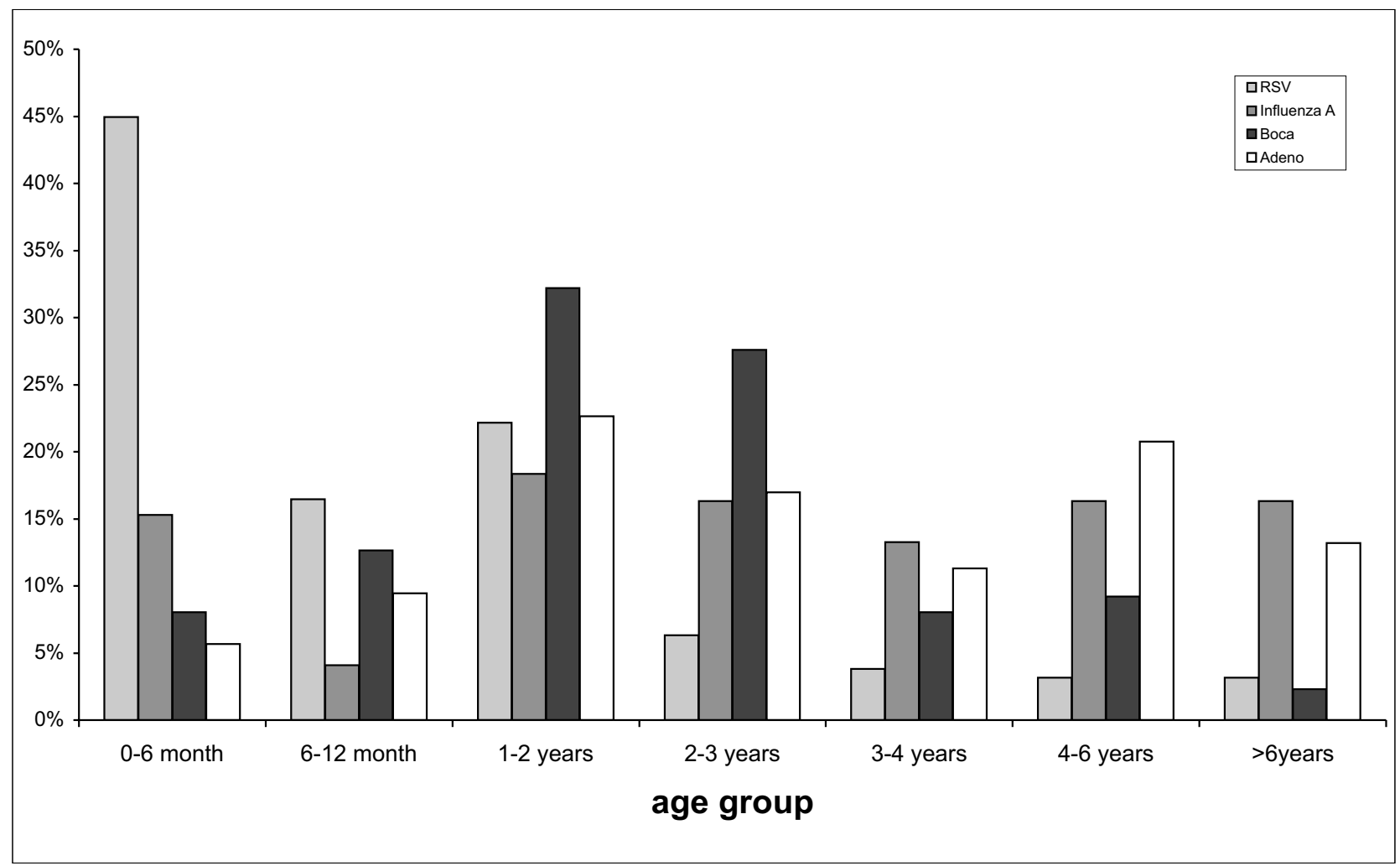

\section{Figure 2}

Age distribution of $\mathrm{hBoV}$ infections. Age distribution of hBoV infected children compared with the age distribution of children infected with RSV, influenza A, or adenoviruses.

methods [10]. Therefore, it is likely that the true prevalence of the respiratory viruses that were analyzed by IFA is actually higher than here reported.

There are several possible explanations for the higher frequency of hBoV infections observed in our study compared to the previous reports. Firstly, the difference may be due to an increased sensitivity of our PCR assay. In all previous studies as well as in ours, single round hot-start PCRs have been employed to detect hBoV DNA. However, the assays vary in the number of PCR cycles performed (35 cycles [3,7-9]; 45 cycles [6]; 50 cycles (present study)).
Depending on the assay optimization, 35 cycles may not be sufficient to detect weakly positive samples. In addition, data on the assay sensitivity were not provided in either of the previous studies. Using a plasmid control, we were able to show that our assay regularly detects approximately 10 copies of hBoV DNA per reaction. In order to obtain information on the amount of hBoV DNA present in the NPAs and in other secretions, a real-time PCR assay is currently under development.

A second potential reason for differing infection frequencies between studies may be due to regional and temporal

Table 2: Median age of patients with viral respiratory tract infections

\begin{tabular}{|c|c|c|c|c|c|}
\hline \multirow[b]{2}{*}{ Infection with } & \multirow[b]{2}{*}{$\mathrm{n}$} & \multirow[b]{2}{*}{ Median age } & \multicolumn{3}{|c|}{ p-values' for groupwise comparison } \\
\hline & & & RSV & Adeno & Influenza A \\
\hline hBoV & 87 & 1.8 & $P<0.0001$ & $P=0.0046$ & $P=0.0031$ \\
\hline RSV & 158 & 0.7 & & $p<0.0001$ & $p<0.0001$ \\
\hline Adenovirus & 53 & 2.7 & & & $p=0.8$ \\
\hline Influenzavirus A & 98 & 2.4 & & & \\
\hline
\end{tabular}

'by Mann-Whitney-test 
Table 3: Seasonal frequency of hBoV infections

\begin{tabular}{ccc}
\hline Period' & NPA samples tested $n$ & hBoV positive NPAs n (\%) \\
\hline Jan 02 - June 02 & 59 & $7(11.9 \%)$ \\
July 02 - June 03 & 159 & $19(11.9 \%)$ \\
July 03 - June 04 & 169 & $19(11.2 \%)$ \\
July 04 - June 05 & 405 & $36(8.9 \%)$ \\
July 05 - Sep 05 & 43 & $6(14.0 \%)$ \\
\hline
\end{tabular}

IObservation periods were chosen from summer to summer whenever possible in order to include the complete winter season

differences in the incidence of $\mathrm{hBoV}$ infection. In contrast to the previous reports, which have studied samples from only one or two winter seasons, the NPAs in our studies have been collected during four consecutive years. In general, seasonal differences of sample acquisition may account for varying incidence numbers. In our study, for example, proportions of influenza A, parainfluenza 3, and adenovirus infections were considerably different between winter seasons (data not shown). However, this was not the case for hBoV infections. In four consecutive winter seasons, we observed a similar frequency of approximately $10 \%$. Therefore, seasonal variation is unlikely to account for the observed high frequency of $\mathrm{hBoV}$ infections in our study population. So far, $\mathrm{hBoV}$ has been detected in Sweden, Australia, Japan, Canada, France, and Germany, and it appears that hBoV has a worldwide distribution. It remains to be determined how incidence numbers are influenced by regional aspects.

Thirdly, the higher frequency observed in our study may be related to different patient populations. Children hospitalized for respiratory tract diseases were included in all studies published so far [3,6-9]. Two studies additionally examined adults and outpatients $[6,8]$. While three studies as well as ours included patients with upper and/or lower respiratory tract disease $[6,8,9]$, two studies focused on patients with lower respiratory tract diseases $[3,7]$.
Thus, it is difficult to compare patient populations in the $\mathrm{hBoV}$ studies.

In agreement with the previous reports $[3,6-9]$, sequencing of the PCR products in the NP-1 region revealed a nucleotide identity of more than $99 \%$ between different samples. This was also true for the two hBoV DNA positive samples from 1998. Although much more sequence information on $\mathrm{hBoV}$ will be required, the data available so far indicate that hBoV may be a highly conserved virus.

The age distribution of $\mathrm{hBoV}$ infections found in our study is similar to previous reports [3,6-9]. Most hBoV infections occurred between 6 months and 3 years of age. This distribution is compatible with protection from infection by maternal antibodies in the first year of life. Future studies of the seroprevalence of $\mathrm{hBoV}$ antibodies in different age groups will shed light on this issue.

Analysis of a potential association between $\mathrm{hBoV}$ infection and clinical manifestations is limited by the retrospective nature of our study, by the high number of double infections, and by the fact that clinical information was obtained only for $\mathrm{hBoV}$ positive patients. However, it seems that there is no obvious association between $\mathrm{hBoV}$ infection and a distinct clinical manifestation. Instead, a broad spectrum of both upper and lower respi-

Table 4: Clinical manifestations in $63 \mathrm{hBoV}$ DNA positive infants and children with and without coinfections ${ }^{1}$

\begin{tabular}{|c|c|c|c|}
\hline Diagnosis at discharge from hospital & $\mathrm{n}$ with coinfection & $\mathrm{n}$ without coinfection & total $\mathrm{n}(\%)$ \\
\hline $\begin{array}{l}\text { Upper respiratory tract disease (rhinitis, otitis media, tonsillitis, pharyngitis, } \\
\text { laryngotracheitis, apnoic spells) }\end{array}$ & 12 & 13 & $25(39.7 \%)$ \\
\hline Lower respiratory tract disease (total) & 8 & 24 & $32(50.8 \%)$ \\
\hline - bronchitis & 3 & 7 & $10(15.9 \%)$ \\
\hline - wheezing bronchitis & 4 & 5 & $9(14.3 \%)$ \\
\hline - bronchiolitis & 0 & 2 & $2(3.2 \%)$ \\
\hline • pneumonia & 1 & $10^{2}$ & II (I7.5\%) \\
\hline Febrile seizures & 3 & 3 & $6(9.5 \%)$ \\
\hline
\end{tabular}

'Clinical information was available for 63 of the $87 \mathrm{hBoV}$ DNA positive samples

${ }^{2} \mathrm{p}=0.044$ by Fisher's exact test 
ratory tract diseases was observed. When clinical diagnoses of hBoV DNA positive patients with and without coinfections were compared, pneumonia was found more frequently in children without coinfection. However, this association of borderline significance $(p=0.044)$ should be regarded with caution because of small numbers. If pneumonia was caused by hBoV infection, it is unclear how a coinfection could result in a less frequent manifestation of this disease.

The assumption in the first description of $\mathrm{hBoV}$, that this virus might be an etiologic agent of respiratory tract disease, was based on the fact that hBoV infections were found significantly more often in samples negative for other respiratory viruses. However, with coinfection rates ranging fom $33.3 \%$ to $55.6 \%$, these findings were neither confirmed by the other studies that analyzed coinfections [6] nor by us. The true number of coinfections in our study is probably even higher than the reported $39.1 \%$, because antigen-based methods were used for screening of respiratory viruses other than $\mathrm{hBoV}$, and because several respiratory pathogens such as coronaviruses, rhinoviruses, enteroviruses and the human metapneumovirus were not tested for. On the basis of the considerable number of coinfections, one might argue that hBoV is an aggravating factor of respiratory diseases, an innocent bystander that is just detected by chance, or a persisting virus that is reactivated by the inflammatory process.

Thus, it is uncertain at present, if hBoV is indeed an etiologic agent of respiratory tract (or other) diseases. Several viruses detected by molecular biology methods in recent years are still in search for a relevant clinical disease $[11,12]$. If hBoV has to be added to this list remains to be determined.

\section{Conclusion}

HBoV is frequently found in NPAs of hospitalized children with acute respiratory tract diseases. Proving the clinical relevance of $\mathrm{hBoV}$ is challenging, because application of some postulates of Koch is not possible. Because of the high rate of coinfections with $\mathrm{hBoV}$ and other respiratory pathogens, an association between $\mathrm{hBoV}$ and respiratory tract diseases remains unproven.

\section{Competing interests}

The author(s) declare that they have no competing interests.

\section{Authors' contributions}

BW and HWK designed and coordinated the study. FN performed the hBoV DNA testing. HWK, KBlessing and KBlath collected clinical data. BW, JS and FT collected virological data. All authors participated in the data anal- ysis. BW and FN drafted the manuscript. All authors read and approved the final version of the manuscript.

\section{Acknowledgements}

We thank the technicians of the viral diagnostic lab for skillful and dedicated assistance and Axel Rethwilm and Kirsty McPherson for helpful comments on the manuscript.

\section{References}

I. Fouchier RA, Rimmelzwaan GF, Kuiken T, Osterhaus AD: Newer respiratory virus infections: human metapneumovirus, avian influenza virus, and human coronaviruses. Curr Opin Infect Dis 2005, I 8(2): $|4|-\mid 46$

2. Woo PC, Lau SK, Chu CM, Chan KH, Tsoi HW, Huang Y, Wong BH, Poon RW, Cai J], Luk WK, Poon LL, Wong SS, Guan Y, Peiris JS, Yuen $\mathrm{KY}$ : Characterization and complete genome sequence of a novel coronavirus, coronavirus HKUI, from patients with pneumonia. J Virol 2005, 79(2):884-895.

3. Allander T, Tammi MT, Eriksson M, Bjerkner A, Tiveljung-Lindell A, Andersson $B$ : Cloning of a human parvovirus by molecular screening of respiratory tract samples. Proc Natl Acad Sci U S A 2005, I 02(36): | 289|- 12896

4. Tattersall P, Bergoin M, Bloom ME, Brown KE, Linden RM, Muzyczka N, Parrish CR, Tijsses P: Family Parvoviridae. In Virus taxonomy: classification and nomenclature of viruses Eighth report of the International Committee on the Taxonomy of Viruses Edited by: Fauquet CM, Mayo MA, Maniloff J, Desselberger U, Ball LA. London, United Kingdom , Elsevier Academic Press; 2005:353-369.

5. Rivers TM: Viruses and Koch's Postulates. Bacteriol 1937, 33(I): $1-12$

6. Sloots TP, McErlean P, Speicher DJ, Arden KE, Nissen MD, Mackay IM: Evidence of human coronavirus HKUI and human bocavirus in Australian children. J Clin Virol 2006, 35(1):99-102.

7. Ma X, Endo R, Ishiguro N, Ebihara T, Ishiko H, Ariga T, Kikuta $H$ : Detection of human bocavirus in Japanese children with lower respiratory tract infections. J Clin Microbiol 2006, 44(3): I I32-II34.

8. Bastien N, Brandt K, Dust K, Ward D, Li Y: Human Bocavirus infection, Canada. Emerg Infect Dis 2006, I 2(5):848-850.

9. Foulongne $V$, Rodiere $M$, Segondy M: Human Bocavirus in children. Emerg Infect Dis 2006, I 2(5):862-863.

10. Herrmann B, Larsson C, Zweygberg BW: Simultaneous detection and typing of influenza viruses $A$ and $B$ by a nested reverse transcription-PCR: comparison to virus isolation and antigen detection by immunofluorescence and optical immunoassay (FLU OIA). J Clin Microbiol 200 I, 39(1): 134-I38.

II. Hino S: TTV, a new human virus with single stranded circular DNA genome. Rev Med Virol 2002, I 2(3): 15 |-158.

12. Stapleton JT: GB virus type C/Hepatitis $\mathbf{G}$ virus. Semin Liver Dis 2003, 23(2): | $37-\mid 48$.

\section{Pre-publication history}

The pre-publication history for this paper can be accessed here:

http://www.biomedcentral.com/1471-2334/6/109/pre pub 